\title{
Optogenetically induced olfactory stimulation in Drosophila larvae reveals the neuronal basis of odor-aversion behavior
}

\section{Dennis Bellmann ${ }^{1}$,Arnd Richardt ${ }^{1}$, Robert Freyberger ${ }^{1}$, Nidhi Nuwal $^{2}$, Martin Schwärzel $^{3}$, André Fiala ${ }^{4}$ and Klemens F. Störtkuhl ${ }^{1 *}$}

\author{
I AG Physiology of Senses, Department of Biology, Ruhr-University Bochum, Bochum, Germany \\ 2 Department of Genetics and Neurobiology, Theodor-Boveri-Institute, Julius-Maximilians-University of Wuerzburg, Wuerzburg, Germany \\ ${ }^{3}$ Department of Biology, University of Berlin, Berlin, Germany \\ ${ }^{4}$ Molecular Neurobiology of Behaviour, Johann-Friedrich-Blumenbach-Institute, Georg-August-University of Goettingen, Goettingen, Germany
}

Edited by:

Martin Giurfa

Université Paul Sabatier - Toulouse III,

France

\section{Reviewed by:}

Thomas Preat, École Supérieure de Physique et de Chimie Industrielles de la Ville de Paris, France Jean-Marc Devaud, CNRS University

Paul Sabatier, France

*Correspondence:

Klemens F. Störtkuhl,

AG-Sinnesphysiologie, Fakultät für

Biologie und Biotechnologie,

Ruhr-Universität Bochum, Gebäude ND

4/30, 44780 Bochum, Germany.

e-mail: klemens.stoertkuhl@rub.de
Olfactory stimulation induces an odor-guided crawling behavior of Drosophila melanogaster larvae characterized by either an attractive or a repellent reaction. In order to understand the underlying processes leading to these orientations we stimulated single olfactory receptor neurons (ORNs) through photo-activation within an intact neuronal network. Using the Gal4UAS system two light inducible proteins, the light-sensitive cation channel channelrhodopsin-2 (ChR-2) or the light-sensitive adenylyl cyclase (Paco) were expressed in all or in individual ORNs of the larval olfactory system. Blue light stimulation caused an activation of these neurons, ultimately producing the illusion of an odor stimulus. Larvae were tested in a phototaxis assay for their orientation toward or away from the light source. Here we show that activation of Pac $\alpha$ expressing ORNs bearing the receptors Or33b or Or45a in blind norpA mutant larvae induces a repellent behavior away from the light. Conversely, photo-activation of the majority of ORNs induces attraction towards the light. Interestingly, in wild type larvae two ligands of Or33b and Or45a, octyl acetate and propionic ethylester, respectively, have been found to cause an escape reaction. Therefore, we combined light and odor stimulation to analyze the function of Or33b and Or45a expressing ORNs. We show that the larval olfactory system contains a designated neuronal pathway for repellent odorants and that activation of a specific class of ORNs already determines olfactory avoidance behavior.

Keywords: Drosophila, olfaction, photo-activation, optogenetics, olfactory behavior, electrophysiology, channelrhodopsin-2, photo-activated adenylyl cyclase

\section{INTRODUCTION}

Odors can be used by animals as cues for their orientation within their environment. Attractive odors that may indicate food sources or mating partners can elicit positive odortaxis, i.e., an orientation and locomotion toward the odor source. Repellent odors such as toxic smells bring forth an opposite response, a negative odortaxis away from the odor source. The Drosophila melanogaster larva represents a favorite model organism to study the principles of olfactory processing in a brain for two reasons. First, its olfactory system is similar to that of vertebrates in its overall anatomical organization but simpler in terms of cell numbers (Vosshall and Stocker, 2007), and even simpler than that of adult flies (Ramaekers et al., 2005; Stocker, 2006; Gerber and Stocker, 2007). Second, chemotaxis of Drosophila larvae is an extremely robust behavior. Most odorants act as attractants for larvae, except for two odorants, octyl acetate and propionic ethylester, which have been discovered so far to function as repellents (Cobb and Dannet, 1994; Cobb, 1999; Heimbeck et al., 1999).

Chemosensation begins with the binding of odorants to olfactory receptors (Ors) expressed in olfactory receptor neurons (ORNs) that are located on the dorsal organs (Vosshall and Stocker, 2007). Two Ors are expressed in each ORN, the non-specific olfactory receptor Or83b that is an ion channel (Sato et al., 2008) and, in addition, a specific Or that determines the response spectrum of the ORN (Störtkuhl and Kettler, 2001; Larsson et al., 2004; Kreher et al., 2005; Neuhaus et al., 2005; Gerber and Stocker, 2007). Each one out of $\sim 25$ specific Ors interacts with a variable number of odorants, and a given odorant can bind to multiple Ors (Kreher et al., 2005). Therefore, the recognition of odorant identity is based on the odorant's combinatorial activation of distinct populations of ORNs, just as it has been described in detail for adult flies (Wang et al., 2003a; Hallem and Carlson, 2006; Hallem et al., 2006). In larvae even the activation of one single ORN is sufficient to elicit positive odortaxis toward a variety of odors, underlining the fact that many odorants are able to activate a given Or (Fishilevich et al., 2005). Whereas the characterization of response spectra of diverse Ors has far advanced (Kreher et al., 2005; Vosshall and Stocker, 2007) the neuronal mechanisms underlying the decision whether an odorant is attractive or repulsive remains unknown for larvae. Aversive or appetitive behavior might be induced in central brain structures based on the processed combinatorial olfactory information, as suggested by Wang et al. (2003b). Alternatively, the decision whether an odorant acts as attractant or repellent might be already determined at the level of individual types of ORNs. This has clearly been shown to be the case in the olfactory system of adult Drosophila (Semmelhack and Wang, 2009), suggesting that also in larvae the attractive or repulsive character of particular odors might be determined already at the level of distinct ORNs. 
However, since now only a pathway for attractive olfactory behavior has been found in mutant anosmic third instar larvae (Fishilevich et al., 2005; Louis et al., 2008) in which only one of the 28 larval Ors was expressed.

Here we have investigated the functional role of individual Ors, not on the basis of their odor specificity, but rather on their capability to trigger positive or negative odortaxis in larvae. To this end we expressed either the light-sensitive cation channel channelrhodopsin-2 (ChR-2) (Schroll et al., 2006) or the photo-activated adenylate cyclase (Pac $\alpha$ ) (Schroder-Lang et al., 2007) in identified ORNs. Illumination causes either a cation-mediated depolarization or a cAMP-mediated activation of these neurons, ultimately creating the illusion of an odor stimulus. We monitored whether larvae were attracted or repelled by the light source. The advantage of this technique relies on the stimulation of single ORNs as components of an otherwise intact neuronal network in the presence of the endogenous Or expression and without using anosmic or deficiency mutants.

\section{MATERIALS AND METHODS FLY STOCKS}

All fly stocks were grown at $24^{\circ} \mathrm{C}$ on standard agar-cornmealmolasses medium with a 12-h light:12-h dark cycle. Transformed flies and their crosses as well as the parental control flies were raised in darkness on food with all-trans retinal (Sigma, Germany). To mix all-trans retinal with the molasses, food was melted and cooled down to $50^{\circ} \mathrm{C}$. From a stock solution of $150 \mathrm{mM}$ all-trans retinal dissolved in 95\% ethanol the appropriate aliquot was mixed with the melted food (Suh et al., 2007). The following fly stocks were described elsewhere: UAS-ChR-2 (Schroll et al., 2006) and UASPac $\alpha$ (Schroder-Lang et al., 2007). Gal4-driver lines (Or13a; Or30a; Or33b; Or35a; Or45a; Or45b; Or59a; Or63a; Or67b; Or74a, Or85c; Or94b) were kindly provided by J. Carlson (Yale University, USA). Gal4-driver Or83b and the anosmic mutant Or83b- were kindly provided by L. Vosshall (Rockefeller University, USA). The wild type (WT) stock was Canton-S. In some experiments we have used blind larvae. In these cases UAS-ChR-2 or UAS-Pac $\alpha$ were homozygous for the norpA P24 mutation that renders the animals blind due to a deletion of a gene encoding a phospholipase $\mathrm{C}$ located on the $\mathrm{X}$-chromosome. Virgins from these lines were crossed with the different Gal4-driver lines. Larvae from this cross were collected from the quadrants after each test to grow them up to adulthood. Subsequently, only blind norpA male flies were analyzed for the tests. The norpA P24 mutant was provided by M. Heisenberg (University of Wuerzburg, Germany). All filial generation of the crosses will be termed according to the driver lines used.

\section{BEHAVIOR TEST}

The larval phototaxis assay and the olfactory test has been described in detail elsewhere (Tompkins, 1979; Lilly and Carlson, 1990). Briefly, third instar larvae were isolated from agar-corn-molasses food with 35\% sucrose in Ringer solution and washed three times with distilled water. For the behavior assays Petri dishes $(90 \mathrm{~mm}$ in diameter) were placed on a partitioned form with two opposing quadrants being transparent and two quadrants being light impermeable. The dishes were coated with $1.5 \%$ agarose to achieve optimal crawling conditions for the larvae. Approximately 20-35 larvae were placed in the center of the dish. For each test the dish was illuminated from the bottom for 2 min either with white or with blue light ( $480 \mathrm{~nm}$ wavelength). The average and overall light intensities measured in the dark quadrants of the dish were $1.1 \mathrm{klx}$ with blue light or $1.135 \mathrm{klx}$ with white light and $20.05 \mathrm{klx}$ or $10.8 \mathrm{klx}$ in the transparent quadrants, respectively (Figure 1A). The intensity of the blue component at $480 \mathrm{~nm}$ wavelength was $0.06 \mathrm{~mW} /$ $\mathrm{cm}^{2}$ for white light and $0.14 \mathrm{~mW} / \mathrm{cm}^{2}$ for the blue light emitting diode. The response index (RI) was calculated by subtracting the number of larvae on the dark quadrants from that on the illuminated quadrants and dividing the difference by the total number of larvae tested. Thus, in a test in which all larvae were repelled by the light the RI would equal -1 . Vice versa, a RI of +1 indicates that all larvae were in the illuminated sections. The olfactory behavior was performed with the same set up in order to allow a comparison of the RI in both tests. Single odorants were presented on $1 \mathrm{~cm}^{2}$ filter papers (Whatman) placed in either the illuminated or in the dark quadrants of the Petri dish. During the test larvae were exposed for 2 min to the odorants. This behavior assay also allowed a simultaneous application of an odor and a light-induced stimulus. In order to differentiate between a negative phototactic reaction and a repellent olfactory response mediated through photostimulation either ChR-2 or Paco expressing larvae were exposed simultaneously to octyl acetate and $480 \mathrm{~nm}$ light (Figure 5). Octyl acetate was applied on small filter papers displayed in the dark quadrants of the Petri dish. After $30 \mathrm{~s}$ light was switched off and the RI was determined (Figure 5A) while the test was running for additional 2 min to allow larvae respond to the octyl acetate. The RI was calculated as explained above (Figure 5B). All tests were performed in darkness.

\section{LARVAL TRACE ANALYSES}

During the behavior test all larvae were recorded with a digital camera (Sony, Japan). The obtained video was projected onto an outline of a Petri dish. Individual traces of larvae were manually drawn and scanned. Traces of all larvae were overlaid using the software CorelDraw (Corel Corporation, USA).

\section{ELECTROPHYSIOLOGICAL RECORDINGS (EDG)}

Electrophysiological recordings from the dorsal organ were generated similar to the technique that was previously used for the electroantennogram (EAG) of adult flies (Störtkuhl and Kettler, 2001). Fixation of the third instar larvae was accomplished according to Kreher et al. (2005) with modifications. Larvae were immobilized on a toothpick and grounded with a silver wire that was wrapped around the toothpick. Alternatively, the wooden toothpick was replaced by a transparent plastic stick when transmitted light was used for the recordings. The recording electrode was positioned on the dome organ under a fluorescence microscopy (Olympus, Japan) and the reference electrode was inserted into the body of the larvae. Odorants were applied using an air flow of 1 1/min directed on the head of the larva. Different odorants and odorant concentrations were prepared in small scintillation vials through which an air stream was guided by an automated custom-built device. Simultaneously, an optical stimulation with blue light $(480 \mathrm{~nm})$ was performed using the fluorescence unit of the microscope. The blue light beam was controlled by an aperture, which allowed 
continuous as well as short time opening of the aperture. To test possible adaptation effects, larvae were exposed for more than $2 \mathrm{~min}$ to $480 \mathrm{~nm}$ light. After $2 \mathrm{~min}$ ethyl acetate was applied for $1 \mathrm{~s}$ and the electrodorsalorganogramm (EDG) measured.

\section{STATISTICS}

To determine statistically significant differences of the RI or $\mathrm{mV}$ values the Student's $t$ test was used. Samples were collected independently and compared pair wise. The statistical evaluation was performed with the software Jump (SAS Instruments, USA). Individual RI values were either generated from 10 or more individual behavioral tests and $\mathrm{mV}$ values electrophysiological recorded form 10 or more individual adult flies or larvae. An analysis of variance (ANOVA) was performed for comparing mutant or transformed lines with the control strain, wild type. When significant differences were found each mutant line was compared with the control line using the Student's $t$ test.

\section{RESULTS}

Larvae show a strong appetitive olfactory behavior towards a variety of odorants (Louis et al., 2008). Only a few odorants have been described to act as repellent substances, among which are ethyl propionate or octyl acetate which are ligands of Or33b and Or45a, respectively, and mediate an escape reaction in the larval olfactory test (Cobb and Dannet, 1994; Hallem et al., 2004; Kreher et al., 2005). The aim of this study was to stimulate single ORNs in the larval olfactory system and to test whether single stimulated ORNs are sufficient for inducing an escape reaction in larvae.

\section{PHOTO-ACTIVATION OF ORNS LEADS TO AN OLFACTORY BEHAVIOR IN LARVAE}

We used the robust negative phototactic behavior of larvae (SawinMcCormack et al., 1995) to address the question if ORNs can be photo-activated in larvae in vivo. Since larvae have an excellent transparency they serve as an ideal system for light-induced stimulation (Schroll et al., 2006). First, wild type larvae were given a choice between an illuminated and a dark quadrant within a Petri dish (Figure 1A). After placing wild type larvae in the center of the dish a very vigorous negative phototactic response was observed. Usually, larvae gather in the non-illuminated areas (Figure 1B). The response index (RI) indicating negative phototaxis after $2 \mathrm{~min}$ was $-0.85(\mathrm{SEM} \pm 0.04)$ when white light was used and -0.86 $(\mathrm{SEM} \pm 0.04)$ for blue light at a wavelength of $480 \mathrm{~nm}$ (Figures 1C and $2 \mathrm{~A}$ ). Video recordings of individual traces showed that wild type larvae approached the border of the dark quadrants but rarely passed into illuminated areas (Figure 1B).

For photo-activation either ChR-2 or Pac $\alpha$ was expressed in all larval ORNs under the control of the ubiquitous Or83b driver (Or83b-Gal4). In this behavioral assay transgenic larvae exhibited a completely different response compared to wild type larvae. Now larvae crossed the border between dark and illuminated partitions of the arena and crawled into the illuminated quadrants (Figure 1C). The RI of ChR-2 or Pac $\alpha$ expressing larvae increased to -0.24 (SEM \pm 0.09$)$ or -0.3 (SEM \pm 0.03$)$, respectively, demonstrating that larvae were now more attracted (or less repelled) by the blue light (Figure 1E). However, since the RI was lower than 0 , larvae were not completely attracted by the light stimulus, most likely because of the repulsive visual stimulus. Yet, under the same test condition RIs of transgenic larvae were statistically different from wild type ( $t$-test, $P<0.05)$. Interestingly, application of white light also induces a repression of the negative phototactic behavior of all transgenic larvae. Expressing ChR-2 or Pac $\alpha$ in all larval ORNs under control of Or83b-Gal4 exhibited a RI of -0.49 $(\mathrm{SEM} \pm 0.07)$ and $\mathrm{RI}-0.4(\mathrm{SEM} \pm 0.06)$ respectively (Figure $1 \mathrm{E})$. RI values of transgenic larvae were statistically different from RI values of wild type larvae at white light. Obviously, white light with a light spectrum that of course includes also $480 \mathrm{~nm}$ is at the used intensity ample to stimulate ChR-2 or Paco. In contrast, parental lines with either the UAS-ChR-2, UAS-Pac $\alpha$ or Gal4-Or83b insertion as well as wild type flies that were fed with retinal to exclude possible influences of retinal on the behavior performed normal negative phototactic behavior indistinguishable from wild type animals. All controls strongly avoided the blue light (Figure 1F), demonstrating the specificity of the lightinduced effects.

\section{LIGHT-INDUCED ORN ACTIVATION DOES NOT PREVENT SIMULTANEOUS ODOR RECOGNITION}

To confirm the behavioral effects on a physiological level we asked whether blue light stimulation is electrophysiologically detectable in ORNs of the dorsal organ. Therefore a recording system to measure field potentials from the dorsal organ was established. According to the EAG widely used in adult flies we name it EDG.

EDG responses as a result of odor stimulation could be recorded with a glass electrode which was placed onto the surface of the dorsal organ. EDG responses that represent ORN sum activity was detectable as a change in voltage amplitude, e.g., for pure ethyl acetate of $12 \mathrm{mV}(\mathrm{SEM} \pm 0.06$; Figure $2 \mathrm{~A})$. In general and in accordance to the low number of ORNs in larvae, responses to all tested odorants were lower as compared to EAGs usually achieved in sum activity recordings in adult wild type flies (Störtkuhl et al., 1999). Interestingly, larvae that express Pac $\alpha$ or ChR-2 in all ORNs stimulation with blue light at the wavelength of $480 \mathrm{~nm}$ showed a similar response of about $10 \mathrm{mV}(\mathrm{SEM} \pm 0.08$; Figure 2B). Evidently the light-activation of either ChR-2 or Pac $\alpha$ leads to a detectable electrophysiological activity in the ORNs within the dorsal organ. However, during the behavioral tests, photo-activation was performed for $30 \mathrm{~s}$. We therefore wanted to know if the light-induced response in ORNs inactivate over time through adaptation processes by stimulation with a long blue light illumination. Therefore larvae that express Pac $\alpha$ in all ORNs were exposed to blue light. After $2 \mathrm{~min}$ of illumination a brief odorant stimulus was given while the light stimulus was still present. We found that an odorant based EDG signal is detectable in addition to the EDG signal that was evoked by $480 \mathrm{~nm}$ light (Figure 2C). In conclusion, ORNs did not strongly adapt during the prolonged blue light stimulation (Figure 2D). The EDG responses (Figure 2D) of either individual stimulations with ethyl acetate in darkness $(6.45 \mathrm{mV}$; SEM $= \pm 0.48)$ or with light only $(5.96 \mathrm{mV}$; SEM $= \pm 0.8)$ were not statistical different from odorant stimulation during a 2 min light stimulation $(5.44 \mathrm{mV}$; SEM $= \pm 0.64$; $t$-test, $P<0.05)$ In other words, the light-induced olfactory stimulus does not prevent the animals from perceiv- 
A

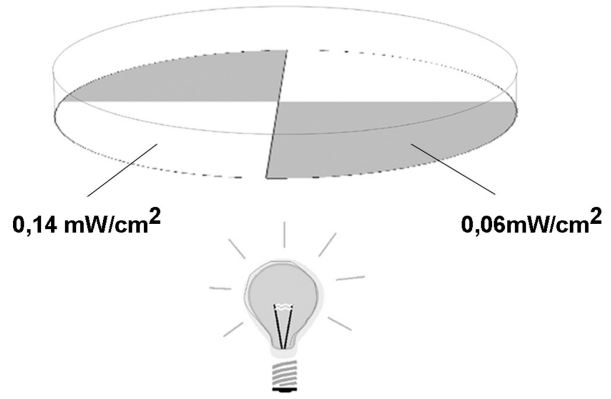

C

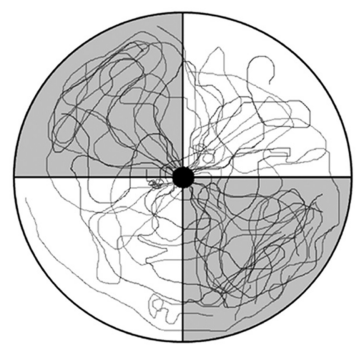

E
B

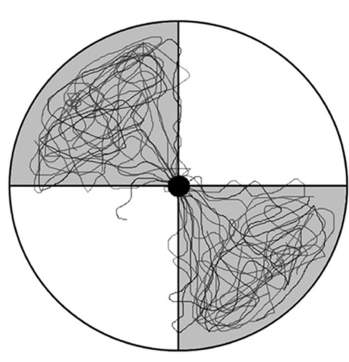

D

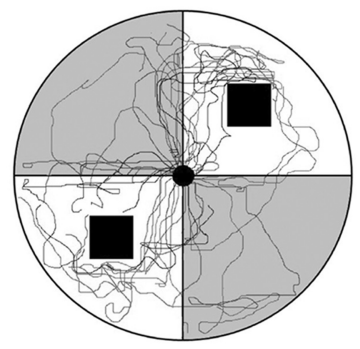

$\mathbf{F}$

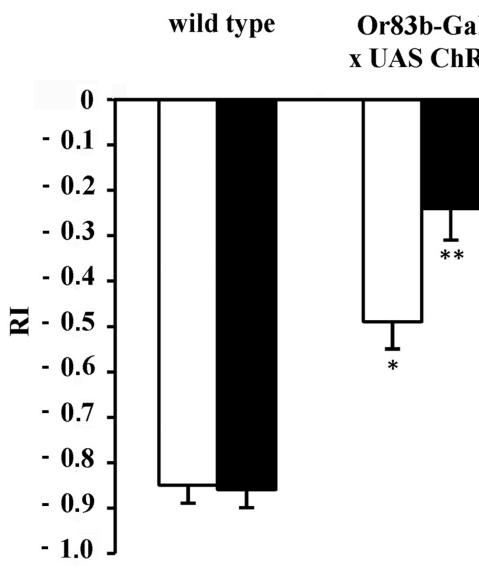

Or83b-Gal4
UAS ChR-2

Or83b-Gal4

$x$ UAS Pac $\alpha$

white light
- blue light

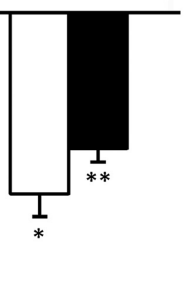

$\bar{x}$

$\bar{\simeq}-0.5$

$-0.6$

$-0.7$

- 0.8

- 0.9 -

$-1.0$ wild type

+ retinal

UAS-

ChR-2

UAS-

Pac $\alpha$

Or83b-

Gal4

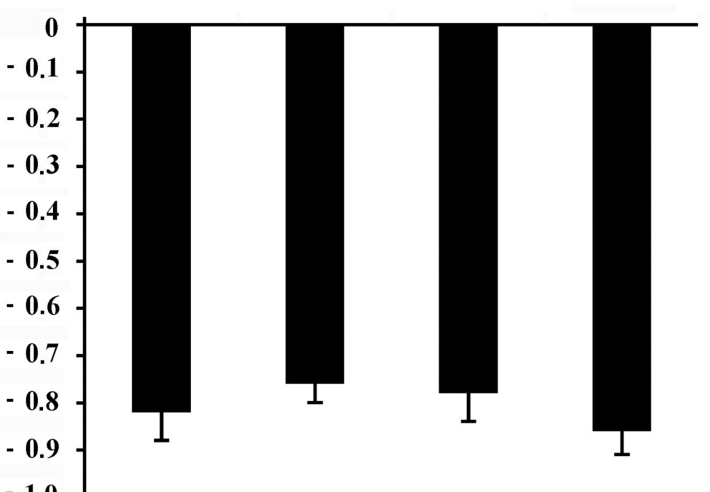

FIGURE 1 | Behavior test assay for larvae. (A) Gray quadrants show dark areas while white sections represent illuminated areas of the Petri dish. Numbers indicate the light intensity at $480 \mathrm{~nm}$. (B) Individual wild type larval crawling traces show negative phototactic reaction to a blue light stimulus $(n=12)$. (C) Traces individual transgenic larvae that express ChR-2 under control of Or83b-Gal4 in all ORNs ( $n=12)$. (D) Traces of wild type larvae $(n=12)$ exposed to benzaldehyde on a filter paper indicated as black squares. (E,F) Response indices (RI) of wild type larvae, transgenic larvae and controls. (E) Wild type and transgenic larvae that express either ChR-2 or Pac $\alpha$ in all ORNs under control of the driver line Or83b-Gal4. ${ }^{*} \mathrm{RI}$ values are statistically different from those of wild type larvae (t-test, $P<0.05$ ); ${ }^{*} \mathrm{RI}$ values are statistically different from those of transformed larvae stimulated with white light ( $t$-test, $P<0.05$ ). Negative phototaxis is strongly reduced in the transgenic strains expressing the light-sensitive proteins in all ORNs. (F) As control strains wild type larvae fed with retinal (wild type + retinal) or parental lines (Or83b-Gal4 and UAS lines) were used ( $n=15$ each). Here, a strong light avoidance can be observed.

\section{OLFACTORY SIGNALS INFLUENCE NEGATIVE PHOTOTACTIC BEHAVIOR}

In summary, for our transgenic larvae expressing the light-sensitive proteins Pac $\alpha$ or ChR-2 in ORNs light serves both as visual signal and as a mimicry of an olfactory signal. In order to interpret the 


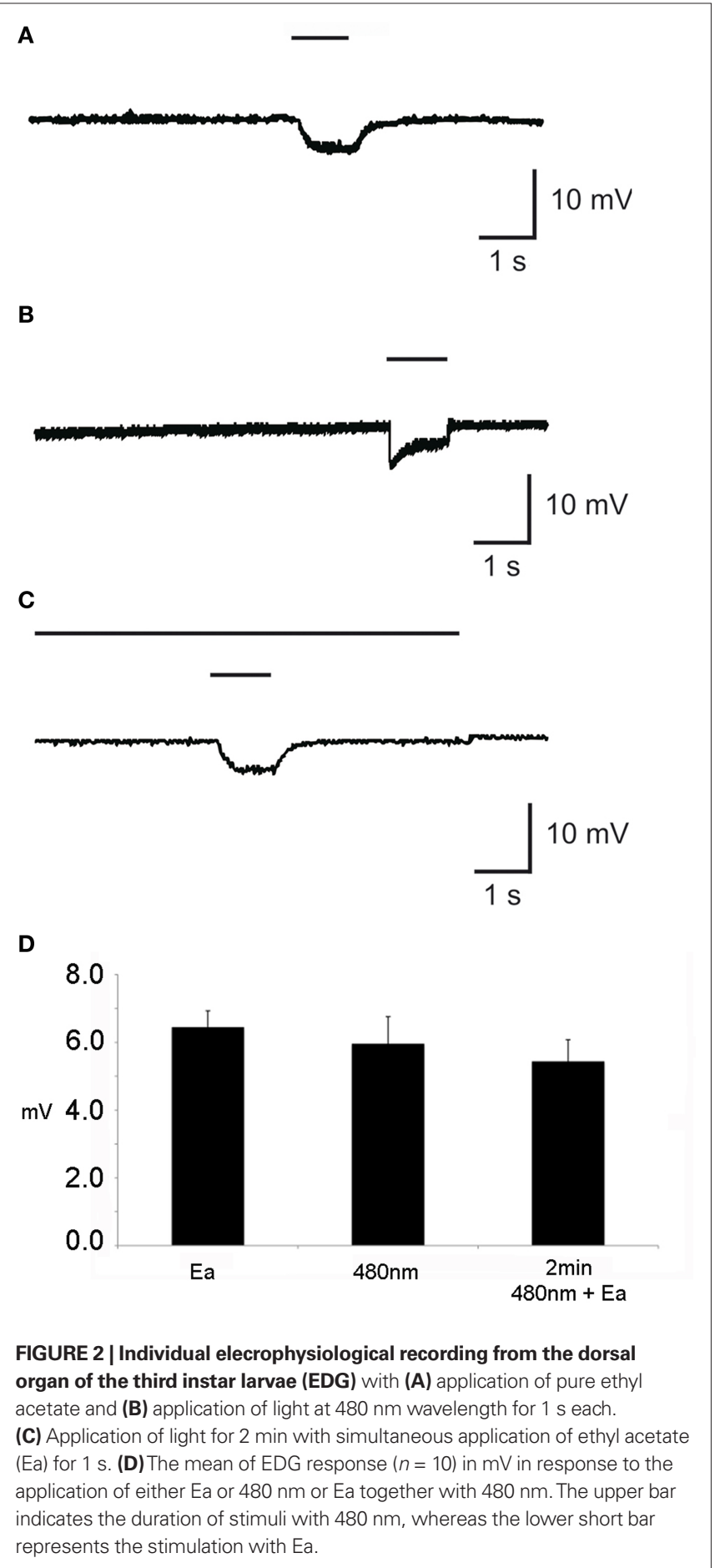

behavioral responses we examined how the visual and the olfactory stimulus interact. In particular we wanted to know whether in wild type larvae the negative phototactic reaction is dominant over an attractive odor stimulus or whether the attractive odor diminishes the visual light avoidance. Therefore we presented a light stimulus and an attractive olfactory stimulus simultaneously. Benzaldehyde is a strong attractant for larvae and was presented at different concentrations in the illuminated quadrants of the Petri dish (Figures $\mathbf{1 D}$ and 3A). Interestingly, under these test

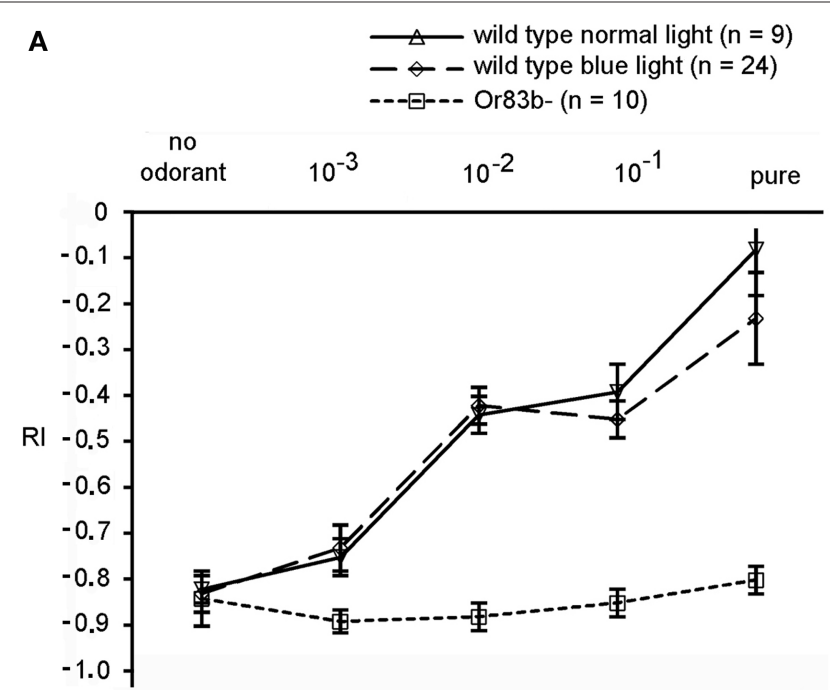

B

$\mathrm{RI}$

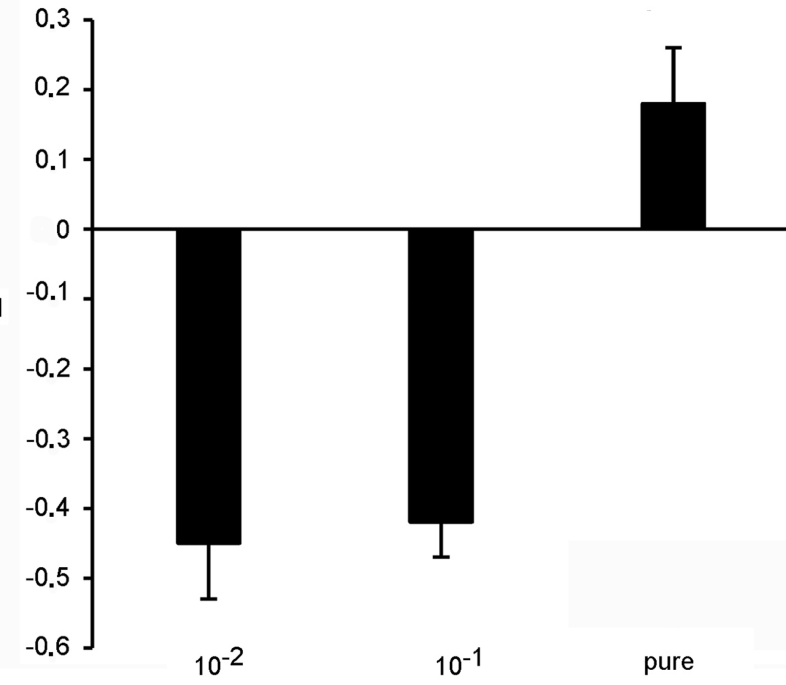

FIGURE 3 | (A) Behavior of wild type larvae exposed to either white or blue light and benzaldehyde in the illuminated quadrants $(n=15)$. The anosmic mutant Or83b- shows only a negative phototactic response, while wild type larvae are more attracted by increasing concentrations of benzaldehyde, as indicated by the decreasing $\mathrm{RI}$ values. (B) Wild type larvae exposed to different concentrations of octyl acetate. With undiluted concentration of octyl acetate applied in the dark quadrants larvae escaped into illuminated sections of the Petri dish. Bars represent means $\pm \operatorname{SEM}(n=15)$.

conditions wild type larvae crawled into the quadrants that were illuminated either with blue or with white light. The behavioral response was dependent on odorant concentration, and larvae got less repelled from blue or normal light by increasing concentrations of benzaldehyde (Figure 3A). In detail, larvae tested with an odorant concentration of 1:100 in paraffin oil (dilution $10^{-2}$ ) had an RI of -0.47 (SEM \pm 0.04 ) with white light and also -0.47 $(\mathrm{SEM} \pm 0.04)$ at $480 \mathrm{~nm}$. In contrast, the anosmic mutant larvae Or83b $\mathrm{b}^{-}$, which are lacking the cation channel Or83b necessary for the functionality of Ors (Larsson et al., 2004; Neuhaus et al., 2005; Sato et al., 2008; Wicher et al., 2008), had low RIs at all tested 
concentrations, confirming that they could not detect the attractive odorant (Figure 3A). Even in the presence of undiluted benzaldehyde the RI was -0.86 ( $\mathrm{SEM} \pm 0.04$ at $480 \mathrm{~nm}$ ), demonstrating a strong negative phototaxis and a complete impairment of the entire olfactory system. In conclusion, the attractive response toward the olfactory stimulus is not prevented by the visual stimulus, i.e., it can to some degree override the repulsive function of light. Conversely, we applied the repellent odorant octyl acetate (Cobb and Dannet, 1994; Cobb, 1999; Heimbeck et al., 1999) in the dark quadrants of the Petri dish and tested if this repellent odor could drive the larvae into the blue light. At undiluted concentrations of octyl acetate wild type larvae prefer to escape into illuminated quadrants of the Petri dish indicated by the positive RI values. Again, this behavior was concentration-dependent. At lower, less repulsive concentrations of this odorant larvae performed a strong negative phototaxis that was more relevant than the olfactory orientation (Figure 3B). This demonstrates that a repellent odorant at high concentration can also override the repulsive effect of strong illumination. These data already indicate that odor stimuli can interact with and influence the behavioral responses to visual stimuli.

\section{PHOTO-ACTIVATION OF INDIVIDUAL ORNs LEADS TO A SPECIFIC OLFACTORY BEHAVIOR}

To elucidate whether photo-activation of individual ORNs evokes a specific olfactory behavior, the driver lines Or33b-Gal4, Or45a-Gal4, Or45b-Gal4, Or59a-Gal4, Or67b-Gal4 Or74a-Gal4, Or85c-Gal4; Gal4-Or94a were used to express either ChR-2 or Pac $\alpha$ in individual ORNs of the larval olfactory system. Moreover, Pac $\alpha$ was also expressed using the driver lines Gal4-Or13a, Gal4Or30a, Gal4-Or35a, and Gal4-Or63a. In general, all transgenic larvae expressing ChR-2 were less attracted by dark quadrants than wild type, confirming that activation of individual ORNs is sufficient to induce odor-evoked behavior (Figure 4A) as already shown by Fishilevich et al. (2005). However, and interestingly, the
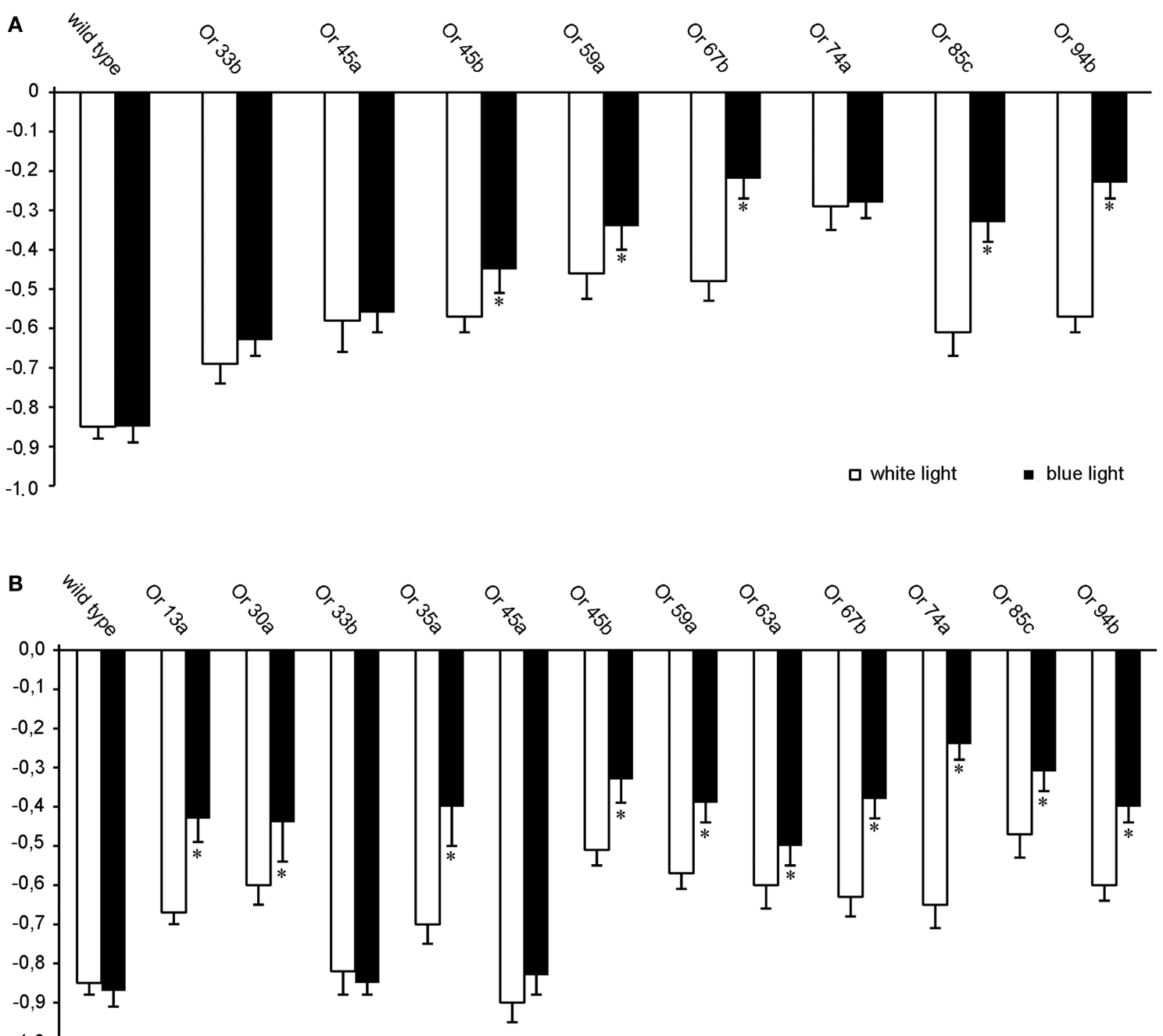

FIGURE 4 | Orientation behavior of transgenic larvae in the four quadrant assay with two quadrants illuminated either with blue or white light, showing the photo-activation of individual ORNs $(n=20)$. Expression of
(A) ChR-2 or (B) Paco in ORNs that express different Ors as indicated. ${ }^{*} \mathrm{RI}$ values are statistically different from $\mathrm{RI}$ values of wild type larvae (t-test, $P<0.05$ ) Bars represent mean \pm SEM. 
stronger effect of blue light compared with white light could not be observed using the driver lines Or33b-Gal4 and Or45a-Gal4. The difference of those two ORN types becomes more obvious when Pac $\alpha$ was expressed (Figure 4B). Here, the negative phototaxis was reduced in all transgenic lines with the exception of Or45a and Or33b (Figure 4B). Photo-activation via Pac $\alpha$ of specific ORNs that endogenously express the odorant receptors either Or45a or Or33b did not induce any change in the RI in response to white light (Figure 4B). Expression of Pac $\alpha$ rather than ChR-2 under the control of the Or45a-Gal4 or Or33b-Gal4 driver resulted in a strong negative phototaxis to blue light with RI of -0.85 (SEM \pm 0.04 ) and RI of -0.82 (SEM \pm 0.06$)$, respectively, which is indistinguishable from the negative phototactic response of wild type larvae to blue light $(\mathrm{RI}=-0.87$; SEM \pm 0.04$)$. We therefore hypothesized that activation of these neurons via an elevation of cAMP levels might be responsible for evoking the negative odortaxis through repellent odorants such as octyl acetate.

\section{PHOTO-ACTIVATION OF Or45a OR Or33b EXPRESSING NEURONS USING PAC $\alpha$ PROVOKES AN AVOIDANCE REACTION}

As shown above, light causes a very strong negative phototactic behavior in Drosophila larvae. Therefore, possible avoidance responses induced by light-dependent activation of ORNs are difficult to identify. A putative light-induced escape reaction of transgenic larvae that express photo-activated proteins under the control of Or33b-Gal4 or Or45a-Gal4 is indistinguishable from the negative phototactic behavior of wild type larvae. In order to clarify whether photo-activation of specific ORNs might cause an olfactory avoidance behavior we applied octyl acetate, a described repellent for larvae (Cobb and Dannet, 1994) as one ligand for Or45a (Kreher et al., 2005) in the non-illuminated quadrants of the Petri dish and simultaneously exposed the opposite quadrants to blue light.

As a control experiment we analyzed the mere reaction to light or octyl acetate. Wild type larvae and all transgenic strains tested performed a normal negative phototactic reaction and avoided the blue light (Figure 5A, black bars). Likewise, all larvae avoided octyl acetate except for the anosmic mutant Or83b- (Figure 5A, white bars). Because octyl acetate is presented in those quadrants opposite to the dark quadrants of the previous experiment the positive RI values shown in Figure 5A indicate the repulsion away from the odorant. However, when the repellent and blue light were applied simultaneously with octyl acetate in the dark quadrants, wild type larvae approached the illuminated quadrants, underlining that the repulsive effect of octyl acetate overrides the repulsive effect of light $(\mathrm{RI}=0.5 ; \mathrm{SEM} \pm 0.08)$. Not surprisingly, anosmic flies show a response indistinguishable from the response evoked by light only. However, flies expressing Pac $\alpha$ in Or45a or Or33b positive neurons, respectively, avoid the illuminated quadrants and stay in the dark quadrants containing octyl acetate $(\mathrm{RI}=-0.68$; SEM \pm 0.04 for Or $45 \mathrm{a}$ and $\mathrm{RI}=-0.25 ; \mathrm{SEM} \pm 0.04$ for Or33b) (Figure $5 \mathrm{~A}$, gray bars). This is what one would expect if the light-activation of the respective neurons would cause a repulsive response. As a control Paco was expressed on Or67b positive neurons. Here, the animals crawl into the illuminated quadrants $(\mathrm{RI}=0.2 ; \mathrm{SEM} \pm 0.08)$ (Figure $5 \mathrm{~A}$, gray bar). Though, when the blue light was subsequently turned off and larvae were allowed to crawl for an additional 2 min they

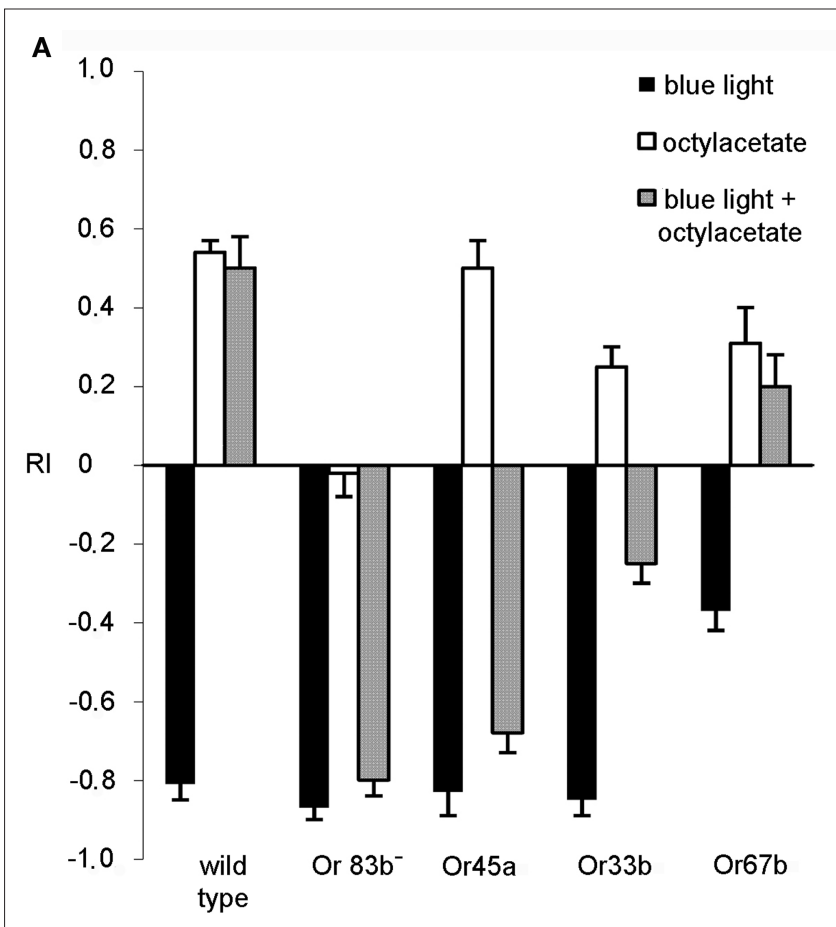

B

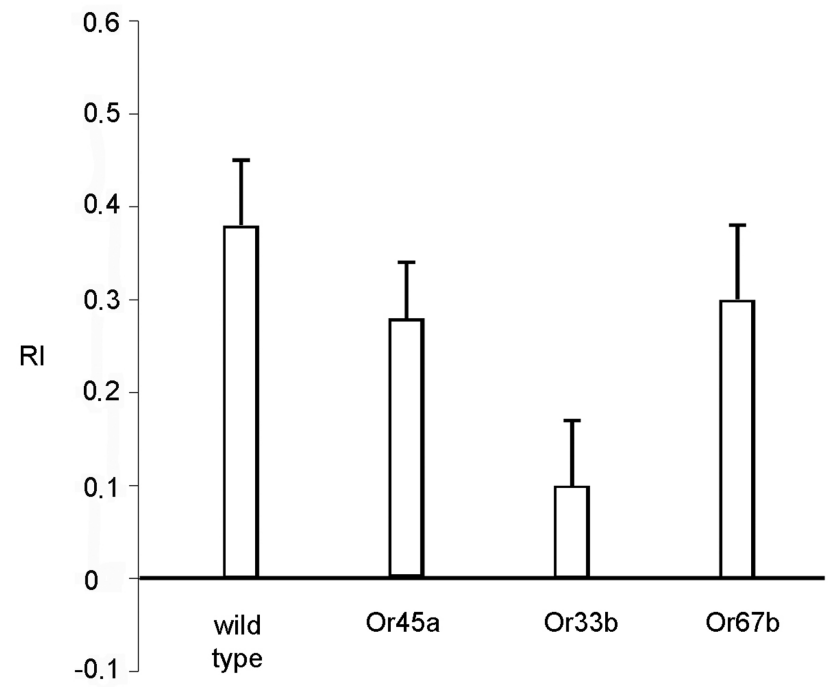

FIGURE 5 | Behavior in the presence of octyl acetate and blue light $(n=10)$. (A) Reaction of wild type, anosmic (Or83b-) and transgenic larvae that express Paco under the control of the indicated driver lines. The repellent octyl acetate was presented on filter papers in the dark areas of the Petri dish. Stimulation was either with octyl acetate (white bars), with blue light (dark bars) or both together over a period of $30 \mathrm{~s}$. (B) The larvae treated with odor and light simultaneously were tested for an additional $2 \mathrm{~min}$ in the presence of octyl acetate in the dark quadrants, but in complete darkness. Bars represent mean \pm SEM.

avoided octyl acetate again (Figure 5B). Within 2 min they crawled into the transparent quadrants of the Petri dish with a RI $=0.28$ $(\mathrm{SEM} \pm 0.06)$ for the Or45a driver and $\mathrm{RI}=0.1(\mathrm{SEM} \pm 0.07)$ for Or33b driver (Figure 5B) indicating that the repellant octyl acetate was predominantly recognized (Figure 5B). The reduced RI values (compare RI values in Figure 5A with Figure 5B) can be explained by the different starting points of individual larvae after 
the light was switched off. Larvae located in dark quadrants have to crawl a longer distance than wild type larvae that are already in the illuminated area in order to escape the repellent applied in the dark section. In conclusion, our experiments demonstrate that the avoidance response caused by the activation of Or45a or Or33b neurons is a highly dominant effect that even overrides the olfactory stimulation of undiluted octyl acetate.

However, the fact that orientation behavior is guided both by olfactory and visual signals make our experimental approach difficult as light-activation of ORNs using ChR-2 or Pac $\alpha$ is confounded by a visual component. To avoid behavioral effects based on inputs from the visual system, we used in addition blind norpA mutant larvae. These larvae carrying an insertion in the norpA gene (P24) lack a phospholipase $\mathrm{C}$ that is required for intact phototransduction (Running Deer et al., 1995). We drove expression of either Paco or ChR-2 in individual ORNs in the norpA mutant background and tested these larvae with the same behavioral assay. Interestingly, norpA mutant larvae were no longer affected by light (Figure 6). Now, blue light served as an olfactory stimulus only. When ChR-2 or Pac $\alpha$ were expressed in all ORNs in the genetic blind norpA background larvae strongly approached the illuminated areas (Figure 6). Interestingly, and in contrast to the expression in all ORNs, when ChR-2 or Pac $\alpha$ were expressed in either Or $45 \mathrm{a}$ or Or33b expressing ORNs only, a clear escape behavior could be observed (Figure 6), showing that the activation of these receptors indeed induced an aversive response.

\section{DISCUSSION \\ PHOTO-ACTIVATION AS A TOOL FOR SPECIFIC ORN STIMULATION IN LARVAE}

We have expressed ChR-2 and Paco in either single or all ORNs of the larval olfactory system and successfully stimulated ORNs through illumination. The substitution of the olfactory cue through a light stimulus did not alter olfactory behavior of transgenic larvae and the robust olfactory behavior through photo-activation was

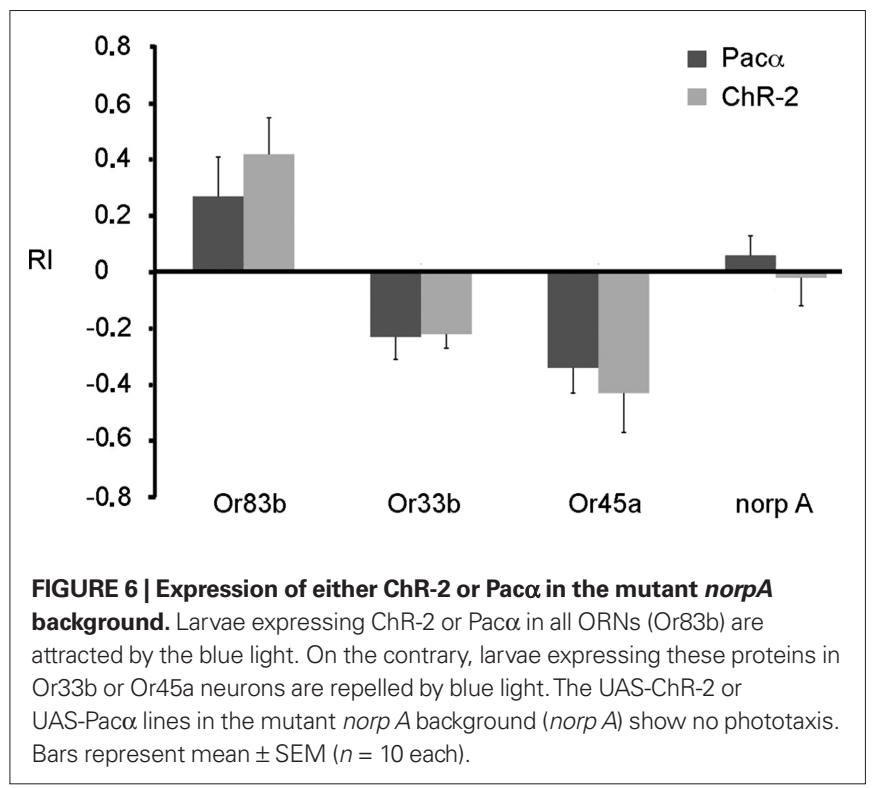

comparable with responses of wild type larvae exposed to odorants. This demonstrates that the expression of these proteins does not cause severe impairments of the neurons under investigation. Also electrophysiologically a response of ORNs expressing these light-sensitive proteins to illumination could be confirmed. From a technical point of view the data show that the photo-activated proteins ChR-2 and Pac $\alpha$ are powerful alternative genetic tools to stimulate ORNs in larvae. The clear advantage of this technique is the non-invasive use of photo-activation which permits a precise stimulation of individual ORNs within an otherwise intact neuronal network. In contrast to other genetically generated larvae used so far, e.g., the genetic ablation of all olfactory neurons and a rescue of single Ors (Fishilevich et al., 2005), this rather new technique requires minimal genetics and therefore does not affect the morphology or physiology of the olfactory system. As photoactivation depends on the transparency of the studied tissue, the larval stage is an ideal system to use this technique, besides its advantages due to the simplicity of its circuitry (Gerber and Stocker, 2007). Furthermore, photo-activated proteins have to match the signal pathway of the neurons. In this regard it is highly interesting that both ChR-2 and Pac $\alpha$ are obviously activating neurons through light to produce a specific olfactory behavior. Whereas the cation channel ChR-2 has been demonstrated to depolarize neurons by illumination (Schroll et al., 2006), our report is the first to show that the light-sensitive adenylyl cyclase Paco can also be used to manipulate ORNs in larval Drosophila. While ChR-2 related cation influx depolarizes neurons directly, the molecular machinery mediating neuronal activation through elevation of cAMP levels has not been uncovered so far. Yet, there are evidences for both IP3 and cAMP dependent olfactory behavior. Mutations affecting these both signal pathways had a quite subtle phenotype (Gomez-Diaz et al., 2004; Wicher et al., 2008). Moreover, olfactory activation of the slow metabotropic pathway produces intracellular cAMP which in turn directly gates Or83b (Wicher et al., 2008). Photo-activation of ORNs through ChR-2 as ionotropic or Pac $\alpha$ as metabotropic pathway is consistent with the finding of a dual activation in other insects such as moths (Zufall and Hatt, 1991). The functional properties of a dual activation concept in ONRs are currently discussed in particular with signal modulation and might extend the range of sensitivity to odors (Nakagawa and Vosshall, 2009). In this respect, it is most interesting that our data clearly demonstrate a role of cAMP in influencing neuronal activity in larval ORNs. It remains to be investigated where exactly in the transduction machinery or upstream of it cAMP might act.

\section{FUNCTIONAL CONTRIBUTION OF SINGLE ORNs TO DISTINCT OLFACTORY BEHAVIOR}

In larvae ORNs form synaptic connections within the larval antennal lobe (LAL) in a non-redundant manner. Each single ORN is connected with one individual glomerulus (Gerber and Stocker, 2007). Subsequently, the stimulation of an individual ORN should lead initially to an activation of a single glomerulus in the LAL. The fact that odorants usually activate multiple receptors to different degrees makes it difficult to determine whether a single ORN already specifies a distinct behavioral output. In addition, processing of olfactory information in the antennal lobe or higher 
brain areas complicates the analysis of neuronal substrates and pathways that determine the ultimate response to a given odor. To overcome this difficulty we have stimulated single ORNs within an otherwise intact olfactory system using photo-activation. Our data confirm that single ORNs contribute to the olfactory behavior (Fishilevich et al., 2005). Light activation may lead to either an olfactory behavior that is comparable to a behavior in the presence of an attractive odorant or to a reaction similar to an avoidance behavior. Similarly, by decomposing the larval olfactory system Fishilevich et al. (2005) found that the stimulation of individual ORNs is sufficient to evoke an olfactory behavior. We conclude from our data that the identity of individual ORNs and their connectivity within a neuronal circuit are more relevant for an olfactory behavior action than the endogenously expressed Or. We therefore suggest a model in which ORNs already define the olfactory preference. Such a model has been proposed in C. elegans (Troemel et al., 1997). Though the organization of the olfactory system is remarkably different in Drosophila, the function of the ORNs is similar to the situation in C. elegans. In detail, individual ORNs in C. elegans express a large number of Ors. Troemel et al. (1997) misexpressed an odorant receptor ODR-10 in an ORN that detects repellants. ODR-10 generates a behavior that is specified by those ORN that expresses ODR-10 endogenously and that detect attractants. In adult Drosophila, one specific ORN has been described that is necessary and sufficient to mediate an escape response, namely the ab1c neuron that expresses Gr21a together with Gr63a (Jones et al., 2007; Suh et al., 2007). In this case, the $\mathrm{ORN}$ is very specifically responsive to the alarm substance $\mathrm{CO}_{2}$. As these ORs deviate from "regular" receptors in their extreme specificity and their ligand $\mathrm{CO}_{2}$ does not cause any combinatorial activity among a range of receptors, this system has been regarded to be the starting point of a designated pathway. Given that larvae might be different from adult flies, we suggest that in fact any olfactory receptor already serves as an inducer of a predetermined response, either attraction or repulsion. Recently, evidence has been reported that supports such a model also for adult flies (Semmelhack and Wang, 2009). It remains to be investigated how the combinatorial activities of these receptors are further integrated into a sum output in behavioral terms.

\section{OLFACTORY STIMULI ARE INFLUENCING THE BEHAVIOR EVOKED BY VISUAL INPUTS IN LARVAE}

We used ChR-2 and Pac $\alpha$ for a combination of photo-activation and odor application as well as for photo-activation of single ORNs in the presence of a given olfactory stimulus. Transgenic and wild type larvae olfactory signals were not behaviorally inhibited by strong and repulsive visual inputs, even when merely a single ORN was activated. This was consistently found in all experimental tests and emphasizes the importance of the olfactory system in larvae. In contrast to the larval situation, many reports show that in adult flies visual inputs seem to have a predominant effect (Helfand and Carlson, 1989; Guo and Götz, 1997). T-maze assays are performed in darkness to avoid visual artifacts during the test. Obviously, during pupal development the hierarchical organization to process simultaneously visual and olfactory information changes and visual cues become more important in adult flies. With respect to the natural environment of larvae that virtually live within their food a dominance of odors versus light appears ecologically plausible.

\section{A SINGLE RECEPTOR CAN MEDIATE AVERSIVE OLFACTORY BEHAVIOR IN LARVAE}

Photo-activation of all larval ORNs including the Or33b and Or45a expressing ORNs leads to an attraction of larvae toward the illuminated quadrants as indicated by the reduced RI value (see Or83bGal4-driver lines; Figure 2). However, photo-activation of a single ORN that expresses endogenously either Or33b or Or45a produces an avoidance behavior induced by blue light or white light (Figures 3C and 5). How can these data be explained? In contrast to adults, larvae show a robust, less concentration dependent olfactory behavior. They are attracted by almost all odorants at most concentrations, except for a few odorants, i.e., octyl acetate and hexyl acetate (Cobb and Dannet, 1994), which are repellents. Based on the repulsive behavior responses through photo-activation of a single ORN our data indicate that in the larvae receptors simply detect odorants while the neurons in which they are expressed direct specific behavioral responses. This also means that in the case of ubiquitous expression of either ChR-2 or Pac $\alpha$ the information of an individual ORN gets more irrelevant. Under this condition different neuronal circuits are stimulated that mediate either attraction or repulsion. These circuits are not independent but process the incoming olfactory information interactively (Fishilevich et al., 2005). Furthermore, larvae detect most odorants as attractants and subsequent appropriate neuronal circuits are predominantly activated might inhibit other neuronal pathways, i.e., neuronal circuits that lead to an avoidance behavior. Lateral inhibition was shown by calcium imaging in the adult antennal lobe of bees (Sachse and Galizia, 2002, 2003) in a dose-dependent manner, in parallel with a recruitment of glomeruli. In addition, excitatory local neurons that lead to a recruitment of olfactory projection neurons, the second-order neurons of the antennal lobe, has been shown for adult flies (Marin et al., 2002; Tanaka et al., 2004; Wilson et al., 2004).

In this respect it is interesting that ChR-2 expression in Or33band Or45a-expressing neurons can cause a reduction in phototactic aversion to light. Cation-mediated depolarization appears to have a different effect than elevation of cAMP levels in these neurons. It remains to be investigated whether this is due to differences in signal transduction within the ORNs or whether ChR-2-mediated activation might be so strong that appetitive pathways are co-activated through the antennal lobe network.

Our data show that photo-activation of single ORNs in an unchanged olfactory network can produce a specific olfactory behavior. Moreover, this technique allows for the combination of olfactory stimulation plus olfactory stimulation mimicked through photo-activation. Interestingly, the repellent ethyl propionate elicits only a very weak electrophysiological response in Or33b expressing ORNs (Cobb and Dannet, 1994; Hallem et al., 2004). Photoactivation of the Or33b expressing ORNs using Paco elicits a very robust escape reaction. Furthermore this receptor is expressed both in the adult and larval stage (Kreher et al., 2005). Based on the unusual odorant specificity, this ORN might have a special warning function in the larval and adult system. It remains to be investigated what the actual "natural" ligands for Or33b and Or 45a might be. 


\section{ACKNOWLEDGMENTS}

We are grateful to A. Balzer-Ferrai for technical support. We thank $\mathrm{V}$. Rodrigues and R. Stocker for reading the manuscript and for valuable suggestions. We are also grateful to Erich Buchner for

\section{REFERENCES}

Cobb, M. (1999). What and how do maggots smell? Biol. Rev. Camb. Philos. Soc. 74, 425-459.

Cobb, M., and Dannet, F. (1994). Multiple genetic control of acetate-induced olfactory responses in Drosophila melanogaster larvae. Heredity 73, 444-455.

Fishilevich, E., Domingos, A. I., Asahina, K., Naef, F., Vosshall, L. B., and Louis, M. (2005). Chemotaxis behavior mediated by single larval olfactory neurons in Drosophila. Curr. Biol. 15, 2086-2096.

Gerber, B., and Stocker, R. F. (2007). The Drosophila larva as a model for studying chemosensation and chemosensory learning: a review. Chem. Senses 32, 65-89.

Gomez-Diaz, C., Martin, F., and Alcorta, E. (2004). The cAMP transduction cascade mediates olfactory reception in Drosophila melanogaster. Behav. Genet. 34, 395-406.

Guo,A., and Gotz,K.G. (1997).Association of visual objects and olfactory cues in Drosophila. Learn Mem. 4, 192-204.

Hallem, E. A., and Carlson, J. R. (2006). Coding of odors by a receptor repertoire. Cell 125, 143-160.

Hallem, E.A., Dahanukar, A., and Carlson, J. R. (2006). Insect odor and taste receptors. Annu. Rev. Entomol. 51, 113-135.

Hallem, E. A., Ho, M. G., and Carlson, J. R. (2004). The molecular basis of odor coding in the Drosophila antenna. Cell 117, 965-979.

Heimbeck, G., Bugnon, V., Gendre, N., Haberlin, C., and Stocker, R. F. (1999). Smell and taste perception in Drosophila melanogaster larva: toxin expression studies in chemosensory neurons. J. Neurosci. 19, 6599-6609.

Helfand, S. L., and Carlson, J. R. (1989). Isolation and characterization of an olfactory mutant in Drosophila with a chemically specific defect. Proc. Natl. Acad. Sci. U.S.A. 86, 2908-2912.

Jones, W. D., Cayirlioglu, P., Kadow, I. G., and Vosshall, L. B. (2007). Two chemosensory receptors together mediate carbon dioxide detection in Drosophila. Nature 445, 86-90.

Kreher, S. A., Kwon, J. Y., and Carlson, J. R. (2005). The molecular basis of odor coding in the Drosophila larva. Neuron 46, 445-456.
Larsson, M. C., Domingos, A. I., Jones, W. D., Chiappe, M. E., Amrein, H., and Vosshall, L. B. (2004). Or83b encodes a broadly expressed odorant receptor essential for Drosophila olfaction. Neuron 43, 703-714.

Lilly, M., and Carlson, J. (1990). smellblind: a gene required for Drosophila olfaction. Genetics 124, 293-302.

Louis, M., Huber, T., Benton, R., Sakmar, T. P., and Vosshall, L. B. (2008). Bilateral olfactory sensory input enhances chemotaxis behavior. Nat. Neurosci. 11, 187-199.

Marin, E. C., Jefferis, G. S., Komiyama, T., Zhu, H., and Luo, L. (2002). Representation of the glomerular olfactory map in the Drosophila brain. Cell 109, 243-255.

Nakagawa, T., and Vosshall, L. B. (2009). Controversy and consensus: noncanonical signaling mechanisms in the insect olfactory system. Curr. Opin. Neurobiol. 19, 284-292.

Neuhaus, E. M., Gisselmann, G., Zhang, W., Dooley, R., Stortkuhl, K., and Hatt, H. (2005). Odorant receptor heterodimerization in the olfactory system of Drosophila melanogaster. Nat. Neurosci. 8, 15-17.

Ramaekers, A., Magnenat, E., Marin, E. C., Gendre, N., Jefferis, G. S., Luo, L., and Stocker, R. F. (2005). Glomerular maps without cellular redundancy at successive levels of the Drosophila larval olfactory circuit. Curr. Biol. 15, 982-992.

Running Deer, J. L., Hurley, J. B., and trol of Drosophila photoreceptor phospholipase C. J. Biol. Chem. 270, 12623-12628.

Sachse, S., and Galizia, C. G. (2002). Role of inhibition for temporal and spatial odor representation in olfactory output neurons: a calcium imaging study. J. Neurophysiol. 87, 1106-1117.

Sachse, S., and Galizia, C. G. (2003). The coding of odour-intensity in the honeybee antennal lobe: local computation optimizes odour representation. Eur. J. Neurosci. 18, 2119-2132.

Sato, K., Pellegrino, M., Nakagawa, T., Vosshall, L. B., and Touhara, K. (2008). Insect olfactory receptors are heteromeric ligand-gated ion channels. Nature 452, 1002-1006.

Sawin-McCormack, E. P., Sokolowski, M. B., and Campos, A. R. (1995). Yarfitz, S. L. (1995). G protein con-

support. This work was supported by grants of the Deutsche Forschungsgemeinschaft (SCHW1410/1-1 to Martin Schwärzel, SFB 554/A2 to André Fiala and STO 283/11-1 to Klemens F. Störtkuhl).

Characterization and genetic analysis of Drosophila melanogaster photobehavior during larval development. J. Neurogenet. 10, 119-135.

Schroder-Lang, S., Schwarzel, M., Seifert, R., Strunker, T., Kateriya, S., Looser, J., Watanabe, M., Kaupp, U. B., Hegemann, P., and Nagel, G. (2007). Fast manipulation of cellular cAMP level by light in vivo. Nat. Methods 4, 39-42.

Schroll, C., Riemensperger, T., Bucher, D., Ehmer, J., Voller, T., Erbguth, K., Gerber, B., Hendel, T., Nagel, G., Buchner, E., and Fiala, A. (2006). Light-induced activation of distinct modulatory neurons triggers appetitive or aversive learning in Drosophila larvae. Curr. Biol. 16, 1741-1747.

Semmelhack, J. L., and Wang, J. W. (2009). Select Drosophila glomeruli mediate innate olfactory attraction and aversion. Nature 459, 218-223.

Stocker, R. F. (2006). Olfactory coding: connecting odorant receptor expression and behavior in the Drosophila larva. Curr. Biol. 16, 16-18.

Stortkuhl, K. F., Hovemann, B. T., and Carlson, J. R. (1999). Olfactory adaptation depends on the Trp Ca2 channel in Drosophila. J. Neurosci. 19, 4839-4846.

Störtkuhl, K. F., and Kettler, R. (2001). Functional analysis of an olfactory receptor in Drosophila melanogaster. Proc. Natl. Acad. Sci. U.S.A. 98, 9381-9385.

Suh, G. S., Ben-Tabou de Leon, S., Tanimoto, H., Fiala, A., Benzer, S., and Anderson, D. J. (2007). Light activation of an innate olfactory avoidance response in Drosophila. Curr. Biol. 17, 905-908.

Tanaka, N. K., Awasaki, T., Shimada, T., and Ito, K. (2004). Integration of chemosensory pathways in the Drosophila second-order olfactory centers. Curr. Biol. 14, 449-457.

Tompkins, L. (1979). Developmental analysis of two mutations affecting chemotactic behavior in Drosophila melanogaster. Dev. Biol. 73, 174-177.

Troemel, E. R., Kimmel, B. E., and Bargmann, C. I. (1997). Reprogramming chemotaxis responses: sensory neurons define olfactory preferences in C. elegans. Cell 91, 161-169.
Vosshall, L. B., and Stocker, R. F. (2007). Molecular architecture of smell and taste in Drosophila. Annu. Rev. Neurosci. 30, 505-533.

Wang, J. W., Wong, A. M., Flores, J., Vosshall, L. B., and Axel, R. (2003a). Two-photon calcium imaging reveals an odor-evoked map of activity in the fly brain. Cell 112, 271-282.

Wang, Y., Chiang, A. S., Xia, S., Kitamoto, T., Tully, T., and Zhong, Y. (2003b). Blockade of neurotransmission in Drosophila mushroom bodies impairs odor attraction, but not repulsion. Curr. Biol. 13, 1900-1904.

Wicher, D., Schafer, R., Bauernfeind, R., Stensmyr, M. C., Heller, R., Heinemann, S. H., and Hansson, B. S. (2008). Drosophila odorant receptors are both ligand-gated and cyclicnucleotide-activated cation channels. Nature 452, 1007-1011.

Wilson, R. I., Turner, G. C., and Laurent, G. (2004). Transformation of olfactory representations in the Drosophila antennal lobe. Science 303, 366-370.

Zufall, F., and Hatt, H. (1991). Dual activation of a sex pheromone-dependent ion channel from insect olfactory dendrites by protein kinase $\mathrm{C}$ activators and cyclic GMP. Proc. Natl. Acad. Sci. U. S. A. $88,8520-8524$.

Conflict of Interest Statement: The authors declare that the research was conducted in the absence of any commercial or financial relationships that could be construed as a potential conflict of interest.

Received: 09 December 2009; paper pending published: 18 January 2010; accepted: 05 May 2010; published online: 02 June 2010.

Citation: Bellmann D, Richardt A, Freyberger R, Nuwal N, Schwärzel M, Fiala A and Störtkuhl KF (2010) Optogenetically induced olfactory stimulation in Drosophila larvae reveals the neuronal basis of odoraversion behavior. Front. Behav. Neurosci. 4:27. doi: 10.3389/fnbeh.2010.00027 Copyright (c) 2010 Bellmann, Richardt, Freyberger, Nuwal, Schwärzel, Fiala and Störtkuhl. This is an open-access article subject to an exclusive license agreement between the authors and the Frontiers Research Foundation, which permits unrestricted use, distribution, and reproduction in any medium, provided the original authors and source are credited. 